Article

\title{
Breast Tumour Kinase (Brk/PTK6) Contributes to Breast Tumour Xenograft Growth and Modulates Chemotherapeutic Responses In Vitro
}

\author{
Rajpal S. Burmi ${ }^{1}$, Gary M. Box ${ }^{2}$, Umar Wazir ${ }^{3}$ (I) , Haroon A. Hussain ${ }^{1}$, Julie A. Davies ${ }^{1, \dagger}$, William J. Court ${ }^{2}$, \\ Suzanne A. Eccles ${ }^{2}$, Wen G. Jiang ${ }^{4}\left(\mathbb{D}\right.$, Kefah Mokbel $^{3}$ and Amanda J. Harvey ${ }^{1, *}$
}

1 Centre for Genome Engineering and Maintenance, Institute for Health Medicine and Environments, Brunel University London, Uxbridge UB8 3PH, UK; rajpal.burmi@gmail.com (R.S.B.); haroon.hussain@brunel.ac.uk (H.A.H.); julie.davies@ge.com (J.A.D.)

2 The Cancer Research UK Cancer Therapeutics Unit, McElwain Laboratories, The Institute of Cancer Research, Sutton SM2 5NG, UK; gary.box@icr.ac.uk (G.M.B.); will.court@icr.ac.uk (W.J.C.); sue.eccles01@icr.ac.uk (S.A.E.)

3 The London Breast Institute, Princess Grace Hospital, London W1U 5NY, UK; umar.wazir@rcsed.ac.uk (U.W.); kefah.mokbel@hcahealthcare.co.uk (K.M.)

4 Cardiff China Medical Research Collaborative, Cardiff University School of Medicine, Heath Park, Cardiff CF14 4XN, UK; jiangw@cardiff.ac.uk

* Correspondence: amanda.harvey@brunel.ac.uk; Tel.: +44-(0)1895-267264

† Now at GE Healthcare, Pollards Wood, Chalfont Saint Giles, Buckinghamshire HP8 4SP, UK.

\section{check for}

updates

Citation: Burmi, R.S.; Box, G.M.;

Wazir, U.; Hussain, H.A.; Davies, J.A.;

Court, W.J.; Eccles, S.A.; Jiang, W.G.;

Mokbel, K.; Harvey, A.J. Breast

Tumour Kinase (Brk/PTK6)

Contributes to Breast Tumour

Xenograft Growth and Modulates

Chemotherapeutic Responses In

Vitro. Genes 2022, 13, 402. https://

doi.org/10.3390/genes13030402

Academic Editor: Vladimir Korinek

Received: 25 January 2022

Accepted: 21 February 2022

Published: 24 February 2022

Publisher's Note: MDPI stays neutral with regard to jurisdictional claims in published maps and institutional affiliations.

Copyright: () 2022 by the authors Licensee MDPI, Basel, Switzerland. This article is an open access article distributed under the terms and conditions of the Creative Commons Attribution (CC BY) license (https:// creativecommons.org/licenses/by/ $4.0 /)$.

\begin{abstract}
Breast tumour kinase (Brk/PTK6) is overexpressed in up to $86 \%$ of breast cancers and is associated with poorer patient outcomes. It is considered a potential therapeutic target in breast cancer, even though the full spectrum of its kinase activity is not known. This study investigated the role of the kinase domain in promoting tumour growth and its potential in sensitising triple negative breast cancer cells to standard of care chemotherapy. Triple negative human xenograft models revealed that both kinase-inactive and wild-type Brk promoted xenograft growth. Suppression of Brk activity in cells subsequently co-treated with the chemotherapy agents doxorubicin or paclitaxel resulted in an increased cell sensitivity to these agents. In triple negative breast cancer cell lines, the inhibition of Brk kinase activity augmented the effects of doxorubicin or paclitaxel. High expression of the alternatively spliced isoform, ALT-PTK6, resulted in improved patient outcomes. Our study is the first to show a role for kinase-inactive Brk in human breast tumour xenograft growth; therefore, it is unlikely that kinase inhibition of Brk, in isolation, would halt tumour growth in vivo. Breast cancer cell responses to chemotherapy in vitro were kinase-dependent, indicating that treatment with kinase inhibitors could be a fruitful avenue for combinatorial treatment. Of particular prognostic value is the ratio of ALT-PTK6:Brk expression in predicating patient outcomes.
\end{abstract}

Keywords: Brk/PTK6; ALT-PTK6; breast cancer; chemotherapy; kinase; prognosis

\section{Introduction}

Breast tumour kinase, also known as protein tyrosine kinase 6 (Brk/PTK6) is overexpressed in a high proportion of invasive breast cancers, and represents a possible target for therapeutic intervention [1,2]. In vitro studies have shown that Brk expression increases breast cancer cell proliferation [3], migration [4-6], and protects the cells from the induction of autophagic and apoptotic cell death [1,7].

Brk involvement in regulating ErbB (erythroblastic leukemia viral oncogene B) receptor signaling is its most well-described role in breast tumorigenesis. Brk is activated in response to ErbB receptor ligands such as EGF or heregulin and, as it associates with all 4 ErbB receptors, Brk has the potential to promote downstream signaling in response to ligand binding [2,8-11]. Increased activation of PI3-K/Akt, p190 RhoGAP, Rac GTPase, Erk5, p38 
MAPK, and ARAP-1 have all been attributed to Brk activity in response to ErbB ligands, resulting in increased cell proliferation, cell cycle progression, and migration [2,11-14].

Brk also regulates processes involved in breast cancer progression in response to non-ErbB receptor ligands such as HGF, OPN, and IGF $[6,7,15]$. Research avenues away from unravelling Brk signaling have focussed on Brk effects in tumour development and treatment.

Despite the wealth of in vitro data and accompanying correlative studies from clinical samples, there are surprisingly few data regarding the potential for increased tumour growth associated with Brk activity. Ptk6 (Brk) expression was shown to enhance the formation of murine ErbB2-induced tumours in cleared fat pads with a shorter latency period than ErbB2-only tumours [12], and both constitutively active and wild-type Brk promoted xenograft growth of MDA-MB-231 basal breast carcinoma cells [16]. However, there are no published data demonstrating kinase dependency in xenograft tumour development.

Xiang and colleagues showed that Brk also decreased the efficacy of the dual EGFR/HER2 inhibitor lapatinib [12]. Our previous work showed that Brk could, in some cellular contexts, affect the relative levels of the anti-apoptotic protein $\mathrm{Bcl}-\mathrm{x}_{\mathrm{L}}$ and its pro-apoptotic splice variant $\mathrm{Bcl}-\mathrm{x}_{\mathrm{S}}$ in favour of $\mathrm{Bcl}-\mathrm{x}_{\mathrm{S}}$ [1]. Given that $\mathrm{Bcl}-\mathrm{x}_{\mathrm{L}}$ has been linked with chemotherapeutic resistance to treatments such as doxorubicin [17], it was hypothesized that Brk expression could modulate tumour cell responses to doxorubicin and possibly other chemotherapeutic agents.

Here we studied whether kinase activity was underlying Brk's growth-promoting effect in human tumour xenografts and breast cancer cell sensitivity to chemotherapeutic agents in vitro and examined whether alternative splicing of Brk had any impact on patient outcomes.

\section{Materials and Methods}

\subsection{Cell Culture}

T-47D breast cancer cells were cultured in RPMI-1640 supplemented with 10\% foetal bovine serum (FBS), $2 \mathrm{mM}$ glutamine, $100 \mathrm{U} / \mathrm{mL}$ penicillin, and $100 \mu \mathrm{g} / \mathrm{mL}$ streptomycin in a humidified atmosphere of $5 \% \mathrm{CO}_{2}$ in air at $37^{\circ} \mathrm{C}$. GI101 cells were cultured in the same medium supplemented with $5 \mu \mathrm{g} / \mathrm{mL}$ insulin.

Brk-transfected cell lines were generated as previously described, with vectors encoding wild-type (WT) or kinase-inactive Brk. Kinase activity was abolished through the mutation of Lysine (K) 219 to Methionine (M) and the protein designated Brk (KM) [1,3]. Cells were maintained in $400 \mu \mathrm{g} / \mathrm{mL}$ G418 (Invitrogen, Paisley, UK).

Paclitaxel-resistant T-47D cells (a gift from Dr Helen Coley at the University of Surrey) were developed by exposing the cells to incrementally increased doses of paclitaxel with each successive passage as previously published for MCF7 cells [18]. Once a resistant phenotype was established, the cells were cultured in RPMI-1640 as above with $4 \mathrm{nM}$ paclitaxel (for resistant cells) or a drug vehicle (for parental cells), at every alternate passage.

\subsection{RNA Interference}

RNA interference protocols were carried out using $120 \mathrm{nM}$ Control non-targeting or PTK6-targeting on-TARGET-PLUS SMART POOL siRNA (Dharmacon, Thermofisher, Hemel Hempsted, UK) and Oligofectamine (Invitrogen, Paisley, UK). A two-hit transfection strategy at $24 \mathrm{~h}$-intervals was employed as previously described [1].

Suppression was confirmed by Western blotting of whole cell protein lysates.

\subsection{Human Tumour Xenografts}

All studies were carried out according to the National Cancer Research Institute (NCRI) guidelines [19].

Xenograft tumours were established by the inoculation of $5 \times 10^{6}$ MDA-MB-157cells, transfected with the empty vector (pRcCMV) or vector encoding a cDNA for either wildtype Brk (WT-Brk) or kinase-inactive Brk (KM-Brk) into the 3rd anterior mammary fat 
pad of female CrTac:NCr-Fox-1(nu) athymic mice. Tumour growth was measured by calipers and volumes were calculated over 118 days. The animals were housed according to institutional and British Home Office regulations in filter boxes in laminar flow cabinets. Food and water, along with all equipment and reagents, was sterilized.

\subsection{Dose Response Determinations}

Cells were seeded at $15 \times 10^{3}$ cells per well into 96-well plates and allowed to adhere overnight. Following serial dilution in vehicle (DMSO or Ethanol) to produce a series of 1000-fold stocks, the drugs were diluted 1:1000 in culture medium and added to the plate at final concentrations between 0.01 and $10 \mu \mathrm{M}$. A 1:1000 dilution of vehicle was used as a control and all wells (control and drug-treated) contained the same volume of vehicle. Drugs were purchased from Sigma- Aldrich (Poole, UK) and Compound $4 \mathrm{f}$ was purchased from Andreas Hilgeroth, Martin-Luther-University Halle-Wittenberg, Universitätsplatz 10, 06108 Halle (Saale)Relative cell numbers were determined after $72 \mathrm{~h}$ of drug treatment by MTT assay (Sigma-Aldrich, Poole, UK).

\subsection{Western Blot}

Whole cell lysates were generated by lysing cells in $2 \times$ SDS-PAGE lysis buffer and proteins were separated by SDS-PAGE on a $10 \%$ SDS-polyacrylamide gel. Following electroblotting in $1 \times$ Towbin buffer onto nitrocellulose membranes, membranes were blocked in $5 \%$ non-fat dried milk in TBS $/ 0.1 \%$ Tween. Membranes were then incubated overnight with a primary antibody diluted 1:10 in 5\% BSA in TBS/0.1\% Tween for Brk (ICR-100) [10] and 1:1000 for $\beta$-actin (Abcam, Cambridge, UK) in 5\% non-fat milk in TBS $/ 0.1 \%$ Tween. Membranes were washed and proteins visualised with an appropriate secondary antibody, conjugated to horseradish peroxidase (Dako, Cambridge, UK) and chemiluminescent substrate (Pierce, Thermofisher, Hemel Hempsted, UK).

\subsection{Human Breast Tumour Samples}

Breast cancer tissues $(n=127)$ and normal background tissue $(n=33)$ were collected and stored at $-80^{\circ} \mathrm{C}$ until the commencement of the study. The patient cohort has been part of a number of completed and on-going studies, and the clinicopathological data describing the patient cohort is further described by Wazir et al. [20]. The Kaplan-Meier survival graphs and statistical analysis were generated using SPSS26. An RUC model was used to generate a cut-off point for grouping the patients into high and low expression groups for survival analysis. Tissue samples were used with informed consent and ethical approval for the experiments were obtained as per institutional guidelines and ethical approval as detailed by Wazir et al. [20].

Statistical analysis was determined at multiple levels using a number of statistical tests, including Logrank (Mantel Cox) for significant difference between high and low PTK6 expression in relation to overall survival at later time points with a Breslow (generalized Wilcoxon) test showing significant difference at the earlier time points. The 100 TaroneWare test was used to further assess the distribution of overall survival with assumptions that there is an identical distribution as well as equal variance.

\subsection{Tissue Processing, RNA Extraction, and cDNA Synthesis}

Approximately $10 \mathrm{mg}$ of cancerous tissue and normal mammary tissue was homogenised and RNA was extracted using an RNA extraction kit from Abgene (Epsom Surrey UK). The concentration of RNA was determined using a UV spectrophotometer (Wolf Laboratories, York, UK) to ensure adequate amounts of RNA for analysis. Reverse transcription was carried out using a reverse transcription kit (AbGene, Epsom, Surrey, UK) with an anchored olig (dT) primer using $1 \mathrm{~g}$ of total RNA in a 96-well plate to produce cDNA. The quality of cDNA was verified using $\beta$-actin primers (primers 5'-ATGATATCGCCGCGCTCGTC-3' and 5'-CGCTCGGTGAGGATCTTCA-3'). 


\subsection{Real-Time PCR}

Quantitative analysis of transcripts of the cDNA library of breast cancer and normal mammary tissues were determined using real-time quantitative PCR based on Amplifluor technology with PCR primers designed using Beacon Designer software (Premier Biosoft International Ltd., Pal Alto, CA, USA) (Table 1). To each of the reverse primers, a Zsequence ( 5 'actgaacctgaccgtaca' 3 ) that was complementary to the Uniprimertm (Millipore) was added and the reaction was carried out using StepOnePlus thermocyclers under the following conditions: $94{ }^{\circ} \mathrm{C}$ for $10 \mathrm{~min}$ and 55 cycles of $94{ }^{\circ} \mathrm{C}$ for $10 \mathrm{~s}, 55^{\circ} \mathrm{C}$ for $30 \mathrm{~s}$, and $72{ }^{\circ} \mathrm{C}$ for 15 with detection of fluorescence signal at the annealing step of each cycle. The levels of each transcript Expression of PTK6 transcripts were normalised against cytokeratin 19 (CK19).

Table 1. Primers used in real-time PCR.

\begin{tabular}{ccc}
\hline Gene & Forward Primer $\left(\mathbf{5}^{\prime} \mathbf{-}^{\prime} \mathbf{3}\right)$ & Reverse Primer $\left(\mathbf{5}^{\prime} \mathbf{-}^{\prime} \mathbf{3}\right)$ \\
\hline PTK6V1 & gttcttggctgcatctc & actgaacctgaccgtacattctcgctgacctgat \\
PTK6V2 & caactacctggccgaga & actgaacctgaccgtacagcctgtggtagttcaca \\
\hline
\end{tabular}

\subsection{Statistical Analysis}

Numerical data was analysed using the Excel spreadsheet software and significance was determined by Student's $t$-test. $p<0.05$ was considered significant.

\section{Results}

\subsection{Brk Promotes Breast Xenograft Formation}

One of the initial aims of this study was to examine the potential of Brk to promote breast tumour growth, independent of exogenous influences such as HER2 expression, to eliminate the effect of HER2 in tumour growth, as most Brk expressing tumours are HER2 negative, and to establish whether the kinase function of Brk was required.

To investigate whether Brk promotes tumour growth, Brk-negative MDA-MB-157 cells, stably transfected with either vector alone, a vector encoding wild-type Brk or kinaseinactive Brk, were injected into mammary fat pads of female CrTac:NCr-Fox-1(nu) athymic mice. Brk expression in MDA-MB-157 was confirmed by Western blotting (Figure 1A). Tumours were allowed to establish and their size was monitored up to 118 days postinjection. Parental MDA-MB-157 cells established small tumours that did not grow above $0.15 \mathrm{~cm}^{2}$ within the time-frame of the study.

The presence of wild-type Brk (WT-Brk) caused a significant increase in the size of tumours compared with the control vector-transfectants (Vector) $(p<0.05)$. Interestingly, kinase-inactive Brk (KM-Brk) also induced a significant increase in tumour volume compared with the vector-only cells $(p<0.05)$ at a rate that was only slightly less than those induced by wild-type Brk. This difference in size between tumours with KM-Brk and WT-Brk was not statistically significant $(p>0.05)$, suggesting that an active kinase domain is not required for xenograft tumour progression (Figure 1B). This opens new questions about the potential of the other domains within Brk that potentiate this growth.

\subsection{Brk Reduces Breast Cancer Cell Susceptibility to Chemotherapeutic Agents In Vitro}

As well as investigating Brk's influence on tumour growth, we were also interested in whether Brk affected tumour cell responses to standard of care chemotherapeutic agents.

The human breast carcinoma cell line T-47D was tested for its responses to a number of chemotherapeutic agents, including doxorubicin and paclitaxel. Suppression of Brk by RNAi (Figure 2A) appeared to sensitise cells to both doxorubicin and Paclitaxel (Figure 2B,C), with the greatest effect seen with doxorubicin. 
A

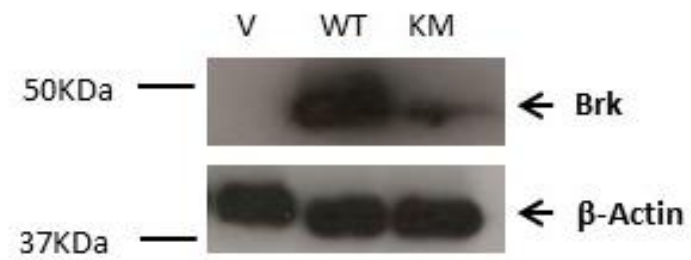

B

Brk expression increases tumour volume in mouse xenografts

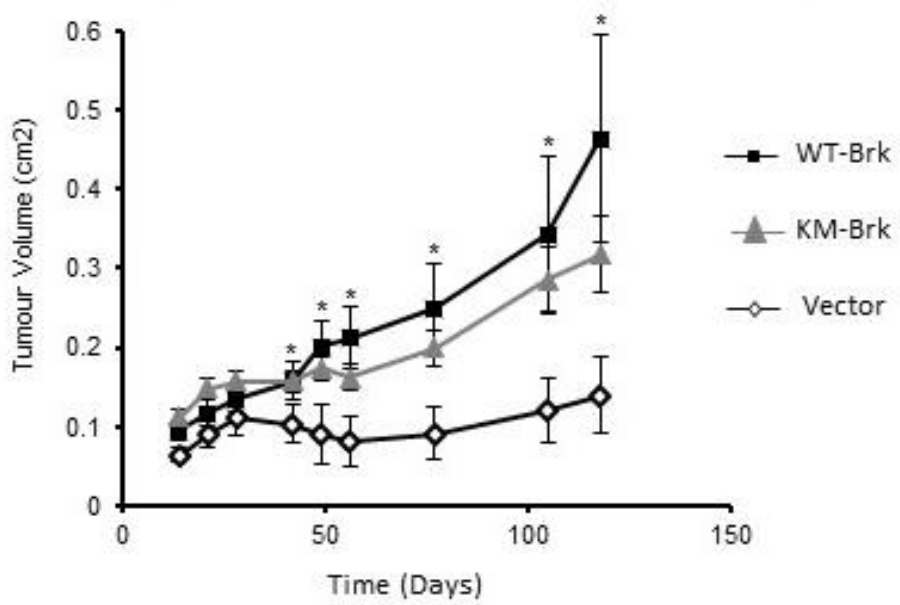

Figure 1. Brk promotes breast tumour xenograft growth (A) MDA-MB-157 cells were stably transfected with either the $\mathrm{pRc} / \mathrm{CMV}$ vector (Vector), or a vector encoding wild-type (WT Brk) or kinaseinactive Brk (KM Brk), and the expression was confirmed by Western blotting with $\beta$-Actin expression as a loading control. (B) Cells were injected into the mammary fat pad of female CrTac:NCr-Fox-1(nu) athymic mice and tumour volume was calculated from the measurement of two perpendicular diameters over 118 days. Mean tumour volume $(\mathrm{n}=10)+/-$ SEM is plotted. * indicates $p<0.05$ (student's $t$-test, WT-Brk compared to the vector control).

When Brk levels were suppressed in the breast cancer cell line GI101 (Figure 2A), the cells again became more susceptible to the effects of paclitaxel or doxorubicin (Figure 2D,E) compared with cells transfected with the control siRNAs. The percentage survival of GI101 cells treated with doxorubicin was significantly reduced at all doses tested between 0.1 and $100 \mathrm{nM}$, and at all doses of paclitaxel tested $(0.1-10 \mu \mathrm{M})$.

In addition, T-47D cells that had been generated to develop resistance to paclitaxel (Tax-R) had elevated Brk levels compared with parental (Par) drug sensitive controls (Supplementary data Figure S1).

To compare the effects of wild-type and kinase-inactive Brk individually on cell sensitivity to chemotherapy agents, a Brk-negative cell line, MDA-MB-468, was selected for transfection. When wild-type Brk was expressed in MDA-MB-468, cells became more resistant to the effects of doxorubicin, but not paclitaxel. Parallel transfections with KM-Brk were also carried out, but this did not affect cellular responses to paclitaxel or doxorubicin compared with vector-only control transfectants (Figure 3A,B). Expression levels were confirmed by Western blotting (Figure 3C).

To confirm that differences in cell growth in response to doxorubicin were not due to an altered proliferative capacity of cells with WT-Brk compared with KM-Brk, the effects of WT-Brk and KM-Brk on proliferation were investigated. Both WT-Brk and KM-Brk promoted the growth of MDA-MB-468 cells under standard culture conditions (Figure 3D), with the KM-Brk-transfected cells reaching higher cell numbers than those transfected with WT-Brk $(p<0.05)$. 

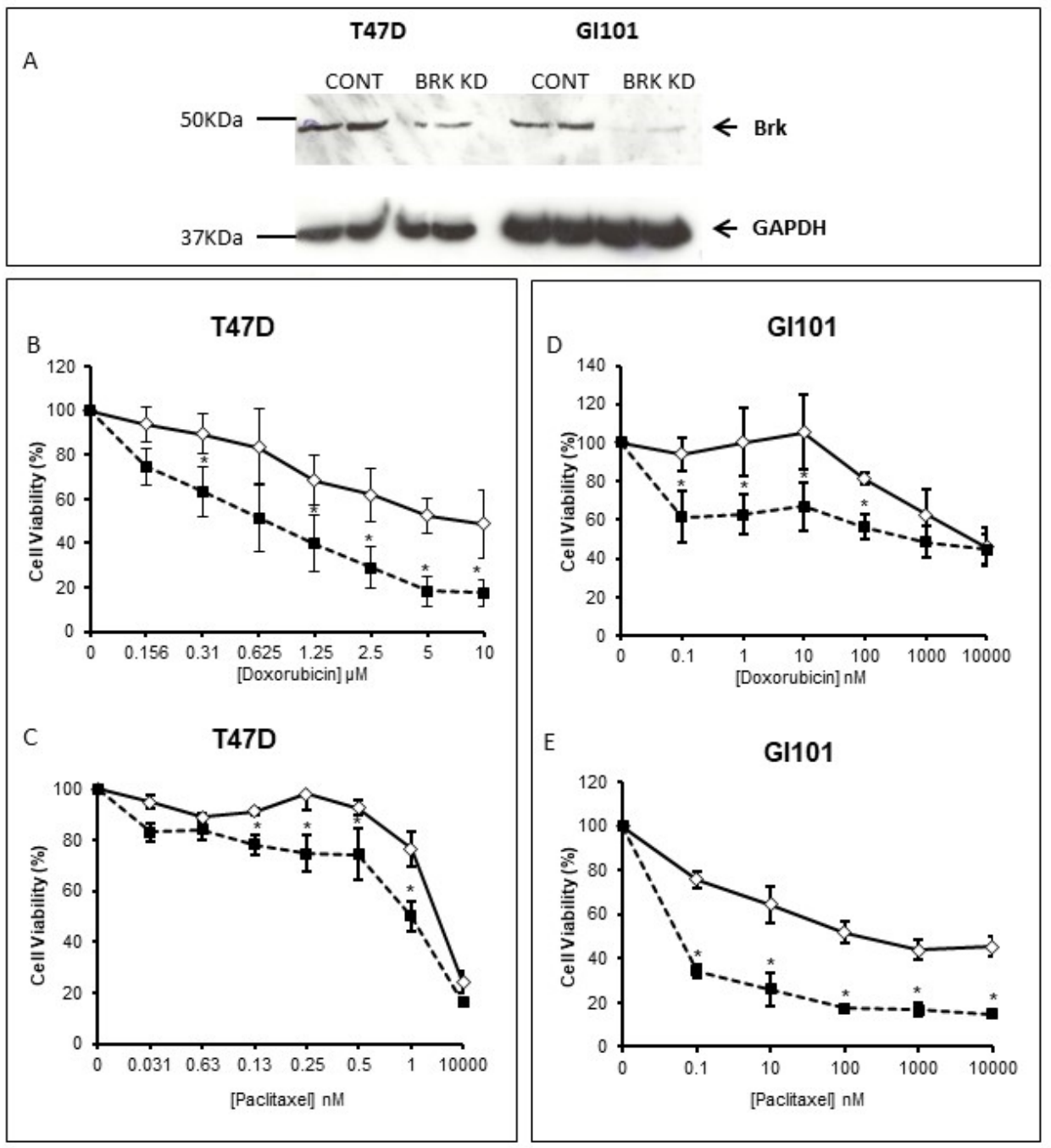

Figure 2. Brk moderates cell responses to chemotherapeutic agents. Brk levels were suppressed in T47D and GI101 cells by RNAi (Brk KD) and suppression was confirmed by Western blotting (A); the cells were seeded and allowed to adhere overnight in 96-well plates. Cells were treated with either doxorubicin (B,D) or Paclitaxel (C,E) for $72 \mathrm{~h}$. Cell viability was determined by MTT assay and expressed as a percentage of the control (vehicle only) wells. The mean \% survival (+/ - SD) of a representative experiment is plotted with the control (transfected with no-target siRNA) in open diamonds and Brk-suppressed cells in closed squares; * indicates $p<0.05$.

Taken together these data suggest that the effects of Brk in mediating cell responses to chemotherapy agents require kinase activity and are not related to proliferative capacity.

\subsection{Brk Inhibition in Triple Negative Breast Cancers}

Due to their lack of receptors/molecular targets, triple negative breast cancers (TNBCs) have more limited treatment options than those that express ER and PR or overexpress HER2 and EGFR, which can be used as targets for therapy.

Given that TNBCs express ptk6 (Brk) (Supplementary Figure S2), that ptk6 (Brk) expression is linked to poorer prognosis in TNBC [21], and Brk has potential as a therapeutic target [22], the effect of Brk kinase inhibition on cell viability was investigated. Inhibition of Brk with the kinase inhibitor compound $4 \mathrm{f}[23,24]$ resulted in significant, but very modest, decreases in cell viability over the dose range used (Figure 4A,D). The effects of $4 \mathrm{f}$ were determined by Western blotting (supplementary Figure S3). 


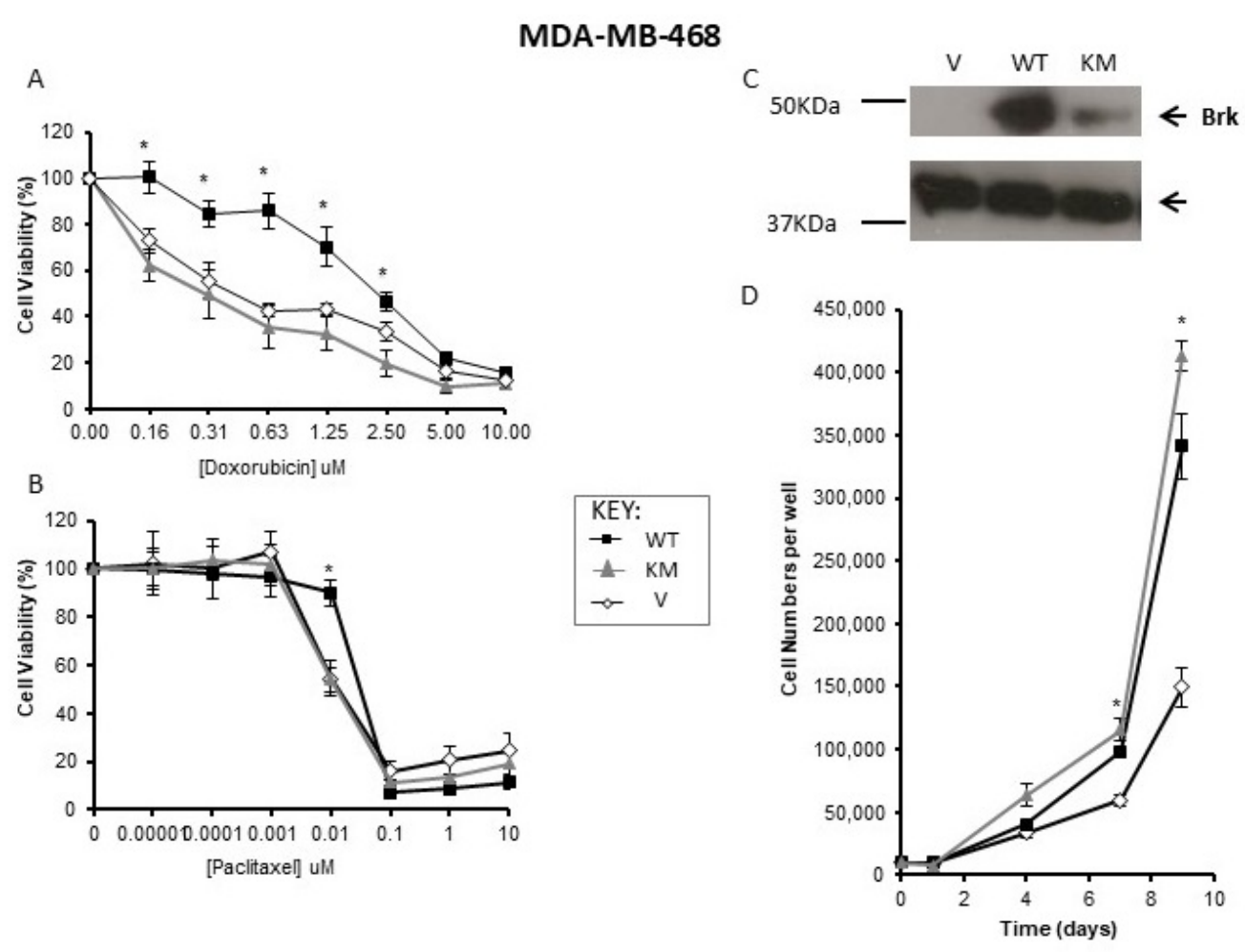

Figure 3. Brk's effects on drug responses are dependent on kinase function and not proliferation. MDA-MB-468 cells, stably transfected with empty vector (V) or constructs expressing either wild-type (WT) or kinase-inactive Brk (KM), were seeded and allowed to adhere overnight in 96-well plates. Cells were treated with either (A) doxorubicin (upper panel) or (B) paclitaxel (lower panel) for $72 \mathrm{~h}$. Cell viability was determined by MTT assay and expressed as a percentage of the control (vehicle only) wells. The mean \% survival (+/-SD) of a representative experiment is plotted; * indicates $p<0.05$. (C) Whole cells lysates were analysed for Brk expression by Western blotting with $\beta$-Actin expression as a loading control. (D) Cells were seeded into 24 well plates and replicate wells counted over 10 days.

Due to the current absence of molecular targets, many patients with TNBC will be treated with chemotherapy, but patients frequently relapse due to intrinsic or acquired resistance to the drugs. The modest decreases in cell viability suggest that inhibition of Brk is unlikely to be a mono-therapy. However, there may be potential to combine Brk inhibition with current standard of care therapeutics such as doxorubicin and paclitaxel. Therefore, the effect of $4 \mathrm{f}$ on TNBC cell responses to paclitaxel and doxorubicin was examined.

Co-treatment with compound $4 \mathrm{f}$ resulted in a modest, but significant, increase in responsiveness to doxorubicin and paclitaxel at the lower doses (nanomolar) in both MDAMB-231 and MDA-MB-436 cells (Figure 4B,C,E,F).

These data suggest that the inhibition of Brk kinase function could improve triple negative breast cancer cell responses to standard of care chemotherapeutic agents.

\subsection{Impact of Brk Splice Variants on Patient Outcomes}

ptk6 mRNA itself is alternatively spliced with the alternate splice variant encoding a truncated protein that lacks the kinase domain and SH2 domains [25]. Given that we have previously shown that $p t k 6$ (Brk) expression has a negative impact on patient outcomes [21], but others have shown Brk to be a positive prognostic indicator, the impact of each isoform on breast cancer patient outcomes was examined. As previously reported, high expression of $p t k 6$ (Brk) was significantly associated with an increasing tumour (TNM) stage $(p=0.02)$ and resulted in reduced overall and disease-free survival (Figure 5A,B). 
MDA-MB-231
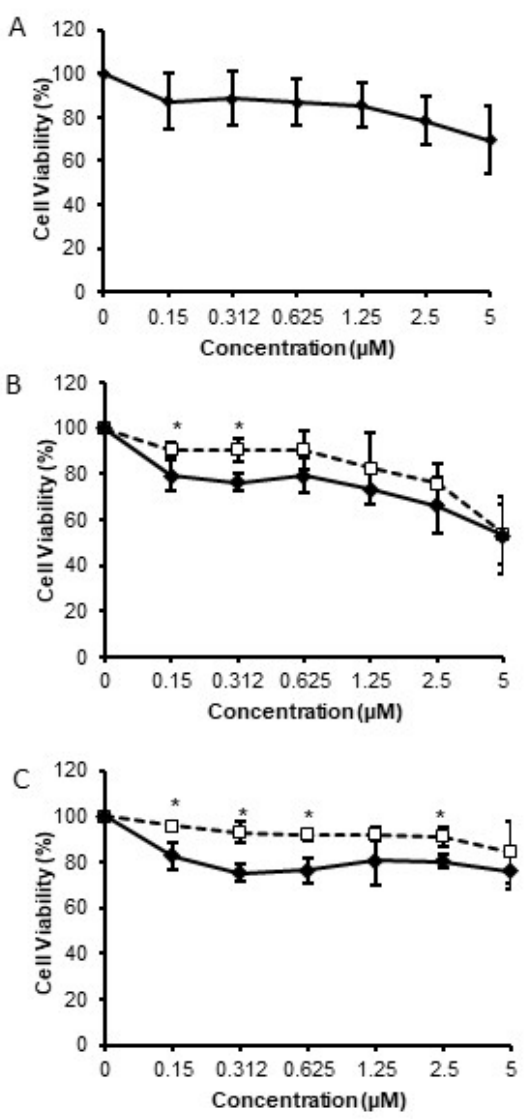

MDA-MB-436

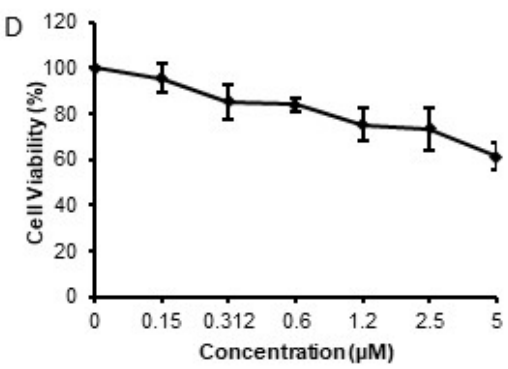

Paclitaxe

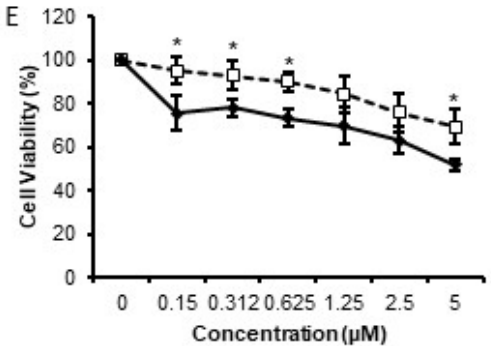

Doxorubicin

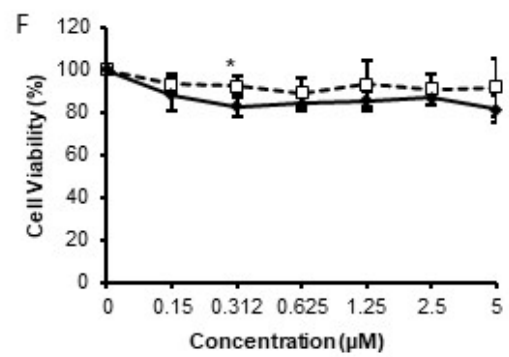

Figure 4. Inhibition of Brk's kinase function increases cell sensitivity to chemotherapeutic agents. Triple negative MDA-MB-231 and MDA-MB-436 cells were seeded and allowed to adhere overnight in 96 wells plates. They were then treated with the Brk inhibitor Compound $4 \mathrm{f}$ alone (A,D) or in combination with paclitaxel $(\mathbf{B}, \mathbf{E})$ or doxrubicin $(\mathbf{C}, \mathbf{F})$ over a range of concentrations $(0-5 \mu \mathrm{m})$. In B-F, open squares represent cells treated with doxorubicin or paclitaxel only; closed diamonds represent cells treated with both $4 \mathrm{f}$ and the chemotherapeutic agent. Cell viability was determined by MTT assay and expressed as a percentage of the control (vehicle only) wells. The mean \% survival $(+/-\mathrm{SD})$ of $\mathrm{n}=3$ experiments is plotted; ${ }^{*}$ indicates $p<0.05$.

A

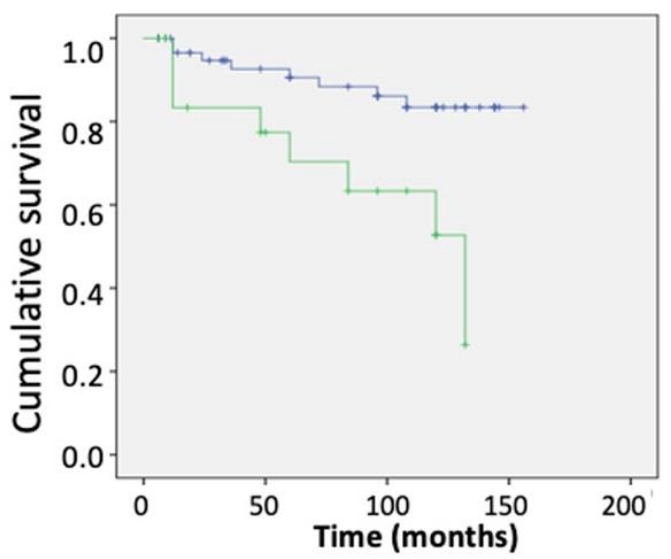

B

Disease-Free Survival

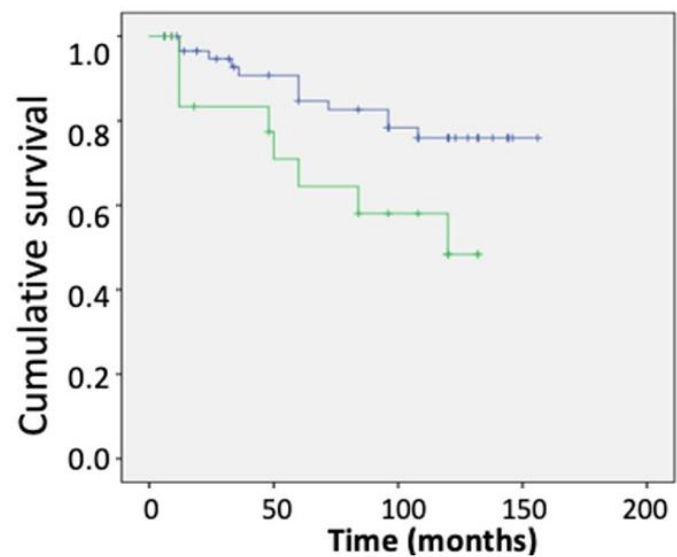

Figure 5. Cont. 

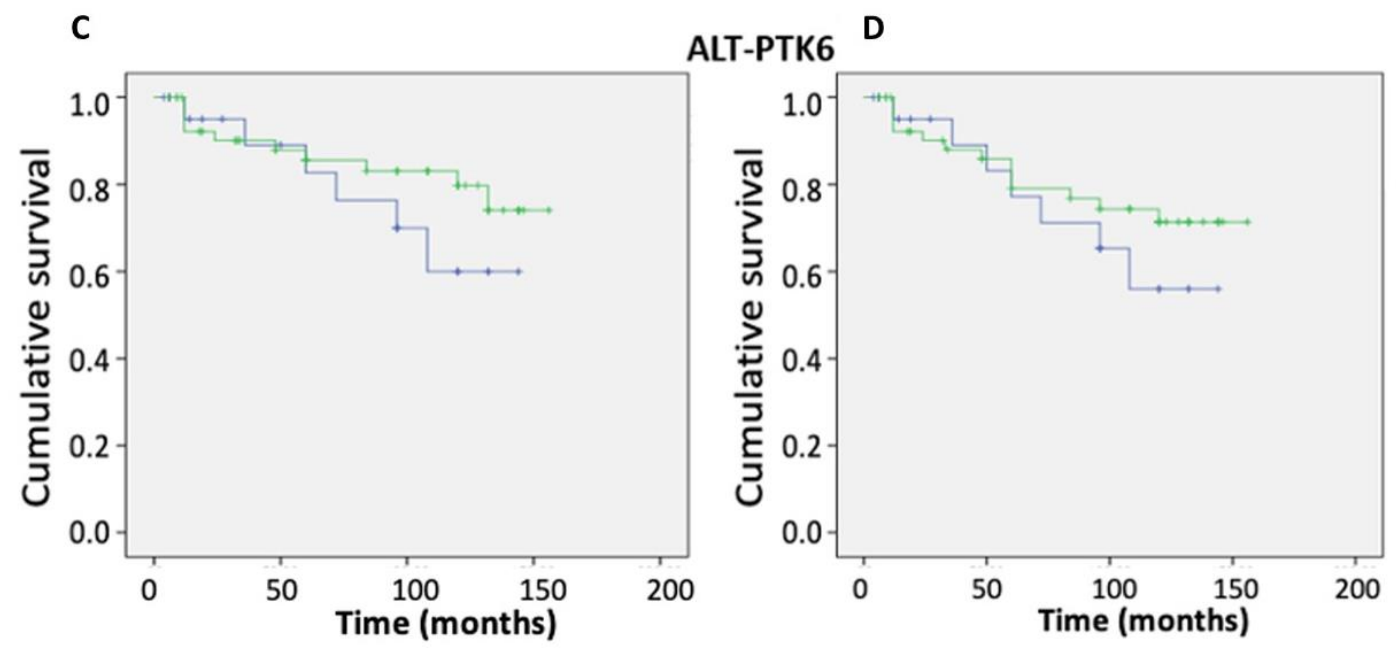

Figure 5. Effect of $p t k 6$ transcripts on patient outcomes. Ptk6 transcript expression was determined in breast cancer tissue $(n=127)$ in relation to the normal background tissue $(n=33)$ using real-time qPCR and correlated with conventional clinic-pathological parameters and clinical outcomes. The Kaplan-Meier curves show high ptk6 expression (green) and low ptk6 (blue) levels in relation to (A) overall and (B) disease-free survival over time, as well as ALT-TPK6 in relation to (C) overall and (D) disease-free survival over time, where high expression is again in green and low expression is in blue. The statistical analysis is shown in the Supplemental Tables S1-S4.

However, the opposite was found for the alternatively spliced isoform ALT-PTK6, where high expression resulted in improved overall and disease-free outcomes (Figure 5C,D). Interestingly, the relative expression of the two isoforms was found to have prognostic significance as a high ratio of ALT-PTK6 expression compared with full length ptk6 transcript also resulted in improved overall survival (Figure 6). When receptor status was taken into account, a high ratio of $p t k 6 /$ ALT-PTK6 transcripts was found in ER-ve tumours (Table 2).

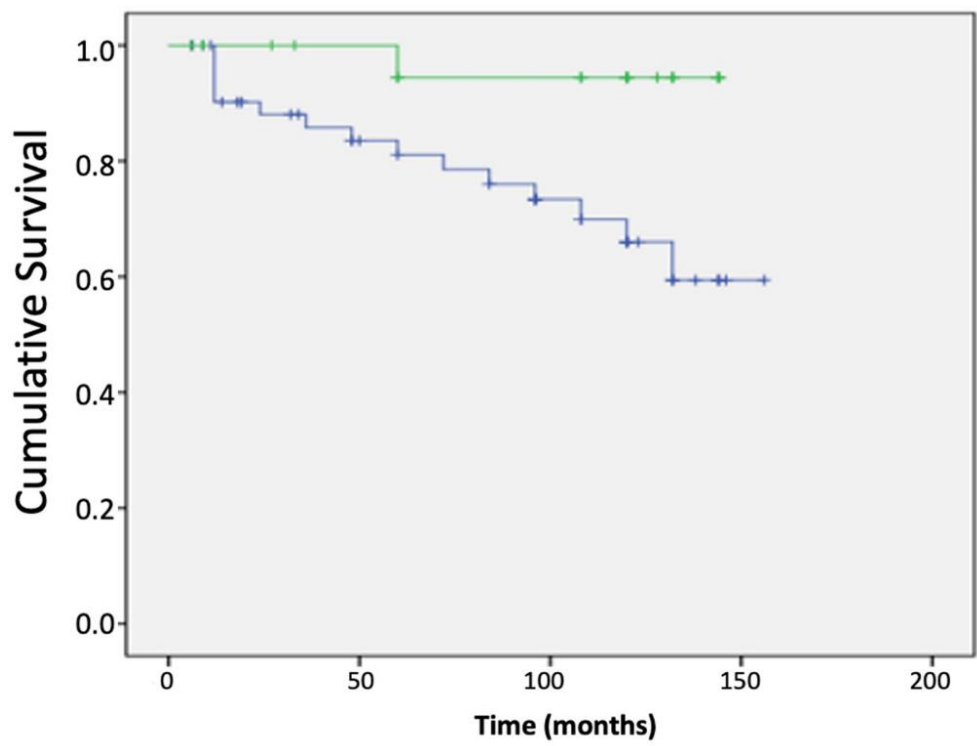

Figure 6. High ALT-PTK6: $p t k 6$ ratio results in favourable overall survival. $p t k 6$ transcript expression was determined in breast cancer tissue $(n=127)$ in relation to the normal background tissue $(n=33)$ using real-time qPCR and correlated with conventional clinic-pathological parameters and clinical outcomes. The Kaplan-Meier curves show high ALT-PTK: ptk6 ratios (green) and low ALT-PTK: ptk6 ratios (blue) levels in relation to overall survival. The statistical analysis is shown in the Supplemental Table S5. 
Table 2. Expression levels of $p t k 6$, ALT-PTK6, and $p t k 6 /$ ALT-PTK6 ratio in relation to hormone receptor status of breast cancer.

\begin{tabular}{|c|c|c|c|c|}
\hline & \multicolumn{2}{|c|}{ HR Status } & Transcript Level (Mean \pm SD) & $p$ Value $^{a}$ \\
\hline \multirow{2}{*}{ ALT-PTK6 } & \multirow{6}{*}{ ER } & $\mathrm{ER}(-)$ & $320.5 \pm 107.0$ & 0.15 \\
\hline & & $\mathrm{ER}(+)$ & $181.9 \pm 68.9$ & \\
\hline & & $\mathrm{ER}(-)$ & $4.6 \pm 2.4$ & 0.262 \\
\hline PTK6 & & $\operatorname{ER}(+)$ & $3.0 \pm 1.6$ & \\
\hline \multirow{2}{*}{ PTK6/ALT-PTK6 ratio } & & $\mathrm{ER}(-)$ & $10,842.3 \pm 5207.3$ & 0.042 \\
\hline & & $\mathrm{ER}(+)$ & $4.5 \pm 4.5$ & \\
\hline \multirow{2}{*}{ ALT-PTK6 } & \multirow{6}{*}{ HER2 } & $\operatorname{HER} 2(-)$ & $274.1 \pm 139.8$ & 0.976 \\
\hline & & $\operatorname{HER} 2(+)$ & $266.4 \pm 78.4$ & \\
\hline & & $\operatorname{HER} 2(-)$ & $3.7 \pm 1.8$ & 0.796 \\
\hline PTK6 & & HER2 (+) & $4.0 \pm 1.9$ & \\
\hline \multirow{2}{*}{ PTK6/ALT-PTK6 ratio } & & $\operatorname{HER} 2(-)$ & $10,885.8 \pm 7001.6$ & 0.616 \\
\hline & & HER2 (+) & $8937.8 \pm 4141.9$ & \\
\hline \multirow{2}{*}{ ALT-PTK6 } & \multirow{6}{*}{ PR } & $\mathrm{PR}(-)$ & $306.1 \pm 90.6$ & 0.2 \\
\hline & & $\mathrm{PR}(+)$ & $168.1 \pm 75.5$ & \\
\hline & & $\mathrm{PR}(-)$ & $4.4 \pm 2.0$ & 0.314 \\
\hline РТК6 & & $\mathrm{PR}(+)$ & $1.4 \pm 0.4$ & \\
\hline \multirow{2}{*}{ PTK6/ALT-PTK6 ratio } & & $\mathrm{PR}(-)$ & $9437.09 \pm 4479.549$ & 0.565 \\
\hline & & $\mathrm{PR}(+)$ & $6507.15 \pm 4462.946$ & \\
\hline
\end{tabular}

${ }^{\mathrm{a}}$ ANOVA test.

\section{Discussion}

The aims of this study were to determine whether the kinase activity of Brk was fundamental for xenograft tumour development, and to investigate a proposed role for Brk in mediating cellular responses to chemotherapeutic agents.

Previous studies have indicated a role for Brk in tumour development alongside ErbB2 overexpression in an immortalized, mouse mammary cell line that retains normal morphology and function in vitro [12,26]. Miah and colleagues (2012) also demonstrated that both wild-type and constitutively active Brk promoted xenograft tumour formation [16]. Our study is the first to show a role for kinase-inactive Brk in human breast tumour xenograft growth. This was striking, but could be interpreted in terms of the multiple roles that Brk has in the processes underlying tumour development [27]. Many of these, for example tumour cell proliferation and migration, do not always require kinase activity $[3,6]$. In addition, abnormal expression of $\beta$-catenin is reported to be one of the mechanisms underlying breast tumorigenesis [28], and Brk is known to regulate $\beta$-catenin in a kinase-independent manner [29]. Interestingly, membrane-targeted Brk activated $\beta$-catenin/TCF-regulated transcription [29], and it is the membrane-localisation that affords Brk its oncogenic properties [30]. It remains to be explored which other Brk domains underlie the xenograft tumour growth, and whether new therapeutics could be developed to target these domains and their substrate interactions.

Our findings contradict those of Jiang and colleagues who reported that inhibiting Brk's kinase function resulted in decreased xenograft growth [31]. It should be noted that the in vivo experiments carried out by Jiang used murine pro BA/F3 B lymphocyte cells, so caution should be exerted when extrapolating their findings to breast cancer.

Brk expression also appeared to regulate breast cancer cell responses to the chemotherapeutic agents paclitaxel and, especially, doxorubicin (Figure 3). As a mitotic spindle regulator, paclitaxel inhibits cell division and promotes cell death by stabilising microtubules in the G2/M phase of the cell cycle [32]. Doxorubicin, on the other hand, inhibits topoisomerase II, and could be predicted to have a greater impact on cell cycle progression from S-phase. Interestingly, previously published data show that Brk suppression results in a decrease in BrdU incorporation in T-47D cells. Parallel experiments examining cell cycle phase showed an increase in the percentage of cells in S-phase when Brk levels were reduced (unpublished findings), suggesting that, although BrdU incorporation decreased, 
Brk-suppression cells entered S-phase, which may make them more susceptible to doxorubicin than paclitaxel. This could account for the apparent difference in the magnitude of responses to doxorubicin and paclitaxel when Brk levels were decreased.

The observed regulation of the responses to chemotherapeutic drugs appeared to be dependent on the kinase activity of Brk (Figure 3). Consistent with our previously published data [3], MDA-MB-468 cells transfected with kinase-active Brk proliferated at a slightly higher rate to those transfected with wild-type Brk (Figure 3C). This suggested that the effect of $p t k 6$ (Brk) expression on drug responses is not an artefact of an increased proliferative response, and is indeed reliant on a functional kinase. Inhibition of Brk by $4 \mathrm{f}$ resulted in a significant, but modest, increase in sensitivity to the chemotherapy agents (Figure 4). The effects seen were weaker than those observed when Brk expression was suppressed by RNAi (Figure 2). This could be explained by the fact that Brk has kinaseindependent functions, especially in relation to cell proliferation [3]. It is also possible that the inhibitor, $4 \mathrm{f}$, is not very potent and a new design of an inhibitor will address this [31]. The effects of $4 \mathrm{f}$ on downstream targets were also very slight (Figure S3). Nonetheless, these data, in conjunction with those from Jiang, provide proof of concept that suppressing Brk kinase activity may enhance chemotherapeutic responses, independent of any effects on tumour progression, and will provide an interesting avenue for future research.

The alternatively spliced variants of Brk had opposing effects on patient outcomes. As expected, based on our previous studies [21], the full length $p t k 6$ transcripts had a negative impact on both overall and disease-free survival. This contrasts markedly with the impact of the ALT-PTK6 variant, which had a positive impact on patient outcomes (Figure 5). Given the truncated isoform lacks both kinase and SH2 domains [25], it is proposed that it acts as a competitive inhibitor of full length Brk [33]. Of particular note is the finding that high PTK6/ALT-PTK6 ratios were associated with ER-ve tumours, i.e., those where treatment options are more limited. Manipulating the expression of the isoforms so that the relative ratio is pushed more towards ALT-ptk6 may have clinical benefit in the longer term.

We, and others, have suggested for some time that inhibiting Brk may be of therapeutic benefit to breast cancer patients but, due to the kinase-independent functions of Brk, this possibility has not been widely pursued. The availability of Brk selective/specific kinase inhibitors remains limited, although there is recent progress in this area with the development of compounds, such as $4 \mathrm{f}$, and the identification of extracts with anti-Brk properties. It is becoming increasingly apparent that targeting Brk with kinase inhibitors in isolation may have clinical limitations, and future Brk-directed therapies may need to focus on the reduction of Brk expression or disruption of protein-protein interactions for therapeutic benefit to be fully realized. This is supported by our data on the xenograft, in which we see growth regardless of the kinase activity of Brk, suggesting that other domains could underlie this growth and could be explored to target Brk alternative functions in the future. Nevertheless, the data in this report suggest that, as part of a multi-drug, combination therapy-based approach, targeting Brk with kinase inhibitors would still be of therapeutic value, especially at lower doses. This approach could have particular significance in Brk-positive, triple negative breast cancers, as they are intrinsically less sensitive to conventional chemotherapy agents and current targeted therapies are of no benefit due to the negative receptor status of the tumours.

\section{Conclusions}

This study is the first to show a role for kinase-inactive Brk in human breast tumour xenograft growth; therefore, it is unlikely that kinase inhibition of Brk, in isolation, would halt tumour growth in vivo. However, we found that breast cancer cell responses to chemotherapy in vitro were kinase-dependent, indicating that treatment with kinase inhibitors could be a fruitful avenue for combinatorial treatment. Determining the relative ratios of Brk isoforms may be needed to fully understand the prognostic significance of Brk expression and/or the implications of any combination therapies. 


\begin{abstract}
Supplementary Materials: The following supporting information can be downloaded at: https: / / www.mdpi.com/article/10.3390/genes13030402/s1. Figure S1: Brk is elevated in Taxol-resistant breast cancer cells. Parental (Parent) and Taxol-resistant (Tax-R) T47D cells were lysed in 2x SDSPAGE loading buffer and Brk levels assessed by western blotting. Figure S2: Triple negative breast cancer (TBNC) cell lines express Brk. Western blot comparing the levels of Brk protein in MDAMB-231 and MDA-MB-436 cells relative to other breast cancer cell lines. Figure S3: Effect of 4f on phosphorylation. MDA-MB-231 (left) and MDA-MB-436 (right) cells were treated with inhibitor $4 \mathrm{f}$ for the indicated time period and the effect on protein levels and phosphorylation determined by western blotting. Table S1: PTK6 overall survival. Table S2: PTK6 disease free survival. Table S3: ALT-PTK6 overall survival. Table S4: ALT-PTK6 disease free survival. Table S5: ALT-PTK6:PTK6 ratio and overall survival.
\end{abstract}

Author Contributions: R.S.B. generated data for Figures 2-4; G.M.B. and W.J.C. did the work presented in Figure 1. J.A.D. contributed to Figure 2; H.A.H. contributed to Figure 3 and generated the data for Figure 4. A.J.H. generated the blots for supplemental data. R.S.B., H.A.H. and J.A.D. were supervised by A.J.H. G.M.B. and W.J.C. were supervised by S.A.E., U.W., W.G.J. and K.M. generated the data in Figure 5. All authors have contributed to the analysis of the data and preparation of the manuscript. All authors have read and agreed to the published version of the manuscript.

Funding: The study was funded by the Breast Cancer Campaign (2006NovPR19 awarded to Amanda J. Harvey), and supported in part by Breast Cancer Hope Foundation.

Institutional Review Board Statement: The study was conducted in accordance with the Declaration of Helsinki, and samples were collected under ethical approval issues by the Bro Taf Health Authority (ethics approval numbers $01 / 4303$ and 01/4046 for ongoing projects). As per the Human Tissue Act (2006), the cDNA libraries fell under the definition of pre-existing holding of acellular samples. The animal study protocol was approved by The Institute of Cancer Research and carried out in accordance with project licence 70/6455 (19b 02).

Informed Consent Statement: Informed consent was obtained from all subjects involved in the study.

Data Availability Statement: No datasets were generated during this study.

Acknowledgments: Germany. Taxol resistant T47D were a gift from Helen Coley, University of Surrey, UK.

Conflicts of Interest: The authors declare no conflict of interest.

\title{
References
}

1. Harvey, A.J.; Pennington, C.J.; Porter, S.; Burmi, R.S.; Edwards, D.R.; Court, W.; Eccles, S.A.; Crompton, M.R. Brk protects breast cancer cells from autophagic cell death induced by loss of anchorage. Am. J. Pathol. 2009, 175, 1226-1234. [CrossRef]

2. Ostrander, J.H.; Daniel, A.R.; Lofgren, K.; Kleer, C.G.; Lange, C.A. Breast tumor kinase (protein tyrosine kinase 6) regulates heregulin-induced activation of erk5 and p38 map kinases in breast cancer cells. Cancer Res. 2007, 67, 4199-4209. [CrossRef]

3. Harvey, A.J.; Crompton, M.R. Use of rna interference to validate brk as a novel therapeutic target in breast cancer: Brk promotes breast carcinoma cell proliferation. Oncogene 2003, 22, 5006-5010. [CrossRef]

4. $\quad$ Chen, H.Y.; Shen, C.H.; Tsai, Y.T.; Lin, F.C.; Huang, Y.P.; Chen, R.H. Brk activates rac1 and promotes cell migration and invasion by phosphorylating paxillin. Mol. Cell. Biol. 2004, 24, 10558-10572. [CrossRef]

5. Lukong, K.E.; Richard, S. Breast tumor kinase brk requires kinesin-2 subunit kap3a in modulation of cell migration. Cell Signal. 2008, 20, 432-442. [CrossRef]

6. Castro, N.E.; Lange, C.A. Breast tumor kinase and extracellular signal-regulated kinase 5 mediate met receptor signaling to cell migration in breast cancer cells. Breast Cancer Res. 2010, 12, R60. [CrossRef]

7. Irie, H.Y.; Shrestha, Y.; Selfors, L.M.; Frye, F.; Iida, N.; Wang, Z.; Zou, L.; Yao, J.; Lu, Y.; Epstein, C.B.; et al. Ptk6 regulates igf-1-induced anchorage-independent survival. PLoS ONE 2010, 5, e11729. [CrossRef]

8. Aubele, M.; Walch, A.K.; Ludyga, N.; Braselmann, H.; Atkinson, M.J.; Luber, B.; Auer, G.; Tapio, S.; Cooke, T.; Bartlett, J.M. Prognostic value of protein tyrosine kinase 6 (ptk6) for long-term survival of breast cancer patients. Br. J. Cancer 2008, 99, 1089-1095. [CrossRef]

9. Aubele, M.; Spears, M.; Ludyga, N.; Braselmann, H.; Feuchtinger, A.; Taylor, K.J.; Lindner, K.; Auer, G.; Stering, K.; Hofler, H.; et al. In situ quantification of her2-protein tyrosine kinase 6 (ptk6) protein-protein complexes in paraffin sections from breast cancer tissues. Br. J. Cancer 2010, 103, 663-667. [CrossRef] 
10. Kamalati, T.; Jolin, H.E.; Mitchell, P.J.; Barker, K.T.; Jackson, L.E.; Dean, C.J.; Page, M.J.; Gusterson, B.A.; Crompton, M.R. Brk, a breast tumor-derived non-receptor protein-tyrosine kinase, sensitizes mammary epithelial cells to epidermal growth factor. J. Biol. Chem. 1996, 271, 30956-30963. [CrossRef]

11. Kamalati, T.; Jolin, H.E.; Fry, M.J.; Crompton, M.R. Expression of the brk tyrosine kinase in mammary epithelial cells enhances the coupling of egf signalling to pi 3-kinase and akt, via erbb3 phosphorylation. Oncogene 2000, 19, 5471-5476. [CrossRef]

12. Xiang, B.; Chatti, K.; Qiu, H.; Lakshmi, B.; Krasnitz, A.; Hicks, J.; Yu, M.; Miller, W.T.; Muthuswamy, S.K. Brk is coamplified with erbb2 to promote proliferation in breast cancer. Proc. Natl. Acad. Sci. USA 2008, 105, 12463-12468. [CrossRef]

13. Shen, C.H.; Chen, H.Y.; Lin, M.S.; Li, F.Y.; Chang, C.C.; Kuo, M.L.; Settleman, J.; Chen, R.H. Breast tumor kinase phosphorylates p190rhogap to regulate rho and ras and promote breast carcinoma growth, migration, and invasion. Cancer Res. 2008, 68, 7779-7787. [CrossRef]

14. Kang, S.A.; Lee, E.S.; Yoon, H.Y.; Randazzo, P.A.; Lee, S.T. Ptk6 inhibits down-regulation of egf receptor through phosphorylation of arap1. J. Biol. Chem. 2010, 285, 26013-26021. [CrossRef]

15. Chakraborty, G.; Jain, S.; Kundu, G.C. Osteopontin promotes vascular endothelial growth factor-dependent breast tumor growth and angiogenesis via autocrine and paracrine mechanisms. Cancer Res. 2008, 68, 152-161. [CrossRef]

16. Miah, S.; Martin, A.; Lukong, K.E. Constitutive activation of breast tumor kinase accelerates cell migration and tumor growth in vivo. Oncogenesis 2012, 1, e11. [CrossRef]

17. Mercatante, D.R.; Mohler, J.L.; Kole, R. Cellular response to an antisense-mediated shift of bcl-x pre-mrna splicing and antineoplastic agents. J. Biol. Chem. 2002, 277, 49374-49382. [CrossRef]

18. Ajabnoor, G.M.; Crook, T.; Coley, H.M. Paclitaxel resistance is associated with switch from apoptotic to autophagic cell death in mcf-7 breast cancer cells. Cell Death Dis. 2012, 3, e260. [CrossRef]

19. Workman, P.; Aboagye, E.O.; Balkwill, F.; Balmain, A.; Bruder, G.; Chaplin, D.J.; Double, J.A.; Everitt, J.; Farningham, D.A.; Glennie, M.J.; et al. Guidelines for the welfare and use of animals in cancer research. Br. J. Cancer 2010, 102, 1555-1577. [CrossRef]

20. Wazir, U.; Ahmed, M.H.; Bridger, J.M.; Harvey, A.; Jiang, W.G.; Sharma, A.K.; Mokbel, K. The clinicopathological significance of lamin a/c, lamin b1 and lamin b receptor mrna expression in human breast cancer. Cell. Mol. Biol. Lett. 2013, 18, 595-611. [CrossRef]

21. Pires, I.M.; Blokland, N.J.; Broos, A.W.; Poujade, F.A.; Senra, J.M.; Eccles, S.A.; Span, P.N.; Harvey, A.J.; Hammond, E.M. Hif1alpha-independent hypoxia-induced rapid ptk6 stabilization is associated with increased motility and invasion. Cancer Biol. Ther. 2014, 15, 1350-1357. [CrossRef] [PubMed]

22. Harvey, A.J.; Crompton, M.R. The brk protein tyrosine kinase as a therapeutic target in cancer: Opportunities and challenges. Anticancer Drugs 2004, 15, 107-111. [CrossRef] [PubMed]

23. Mahmoud, K.A.; Krug, M.; Wersig, T.; Slynko, I.; Schachtele, C.; Totzke, F.; Sippl, W.; Hilgeroth, A. Discovery of 4-anilino alpha-carbolines as novel brk inhibitors. Bioorg. Med. Chem. Lett. 2014, 24, 1948-1951. [CrossRef] [PubMed]

24. Oelze, M.; Mahmoud, K.A.; Sippl, W.; Wersig, T.; Hilgeroth, A.; Ritter, C.A. Novel 4-anilino-alpha-carboline derivatives induce cell death in nonadhesive breast cancer cells through inhibition of brk activity. Int. J. Clin. Pharmacol. Ther. 2015, 53, 1052-1055. [CrossRef] [PubMed]

25. Mitchell, P.J.; Barker, K.T.; Shipley, J.; Crompton, M.R. Characterisation and chromosome mapping of the human non receptor tyrosine kinase gene, brk. Oncogene 1997, 15, 1497-1502. [CrossRef]

26. Danielson, K.G.; Oborn, C.J.; Durban, E.M.; Butel, J.S.; Medina, D. Epithelial mouse mammary cell line exhibiting normal morphogenesis in vivo and functional differentiation in vitro. Proc. Natl. Acad. Sci. USA 1984, 81, 3756-3760. [CrossRef]

27. Harvey, A.; Burmi, R. Future therapeutic strategies: Implications for brk targeting. In Breast Cancer Current and Alternative Therapeutic Modalities; Gunduz, E., Gunduz, M., Eds.; InTech: London, UK, 2011; pp. 413-434.

28. Wang, S.; Li, W.; Lv, S.; Wang, Y.; Liu, Z.; Zhang, J.; Liu, T.; Niu, Y. Abnormal expression of nek2 and beta-catenin in breast carcinoma: Clinicopathological correlations. Histopathology 2011, 59, 631-642. [CrossRef]

29. Palka-Hamblin, H.L.; Gierut, J.J.; Bie, W.; Brauer, P.M.; Zheng, Y.; Asara, J.M.; Tyner, A.L. Identification of beta-catenin as a target of the intracellular tyrosine kinase ptk6. J. Cell Sci. 2010, 123, 236-245. [CrossRef]

30. Ie Kim, H.; Lee, S.T. Oncogenic functions of ptk6 are enhanced by its targeting to plasma membrane but abolished by its targeting to nucleus. J. Biochem. 2009, 146, 133-139. [CrossRef]

31. Jiang, J.; Gui, F.; He, Z.; Li, L.; Li, Y.; Li, S.; Wu, X.; Deng, Z.; Sun, X.; Huang, X.; et al. Targeting brk-positive breast cancers with small-molecule kinase inhibitors. Cancer Res. 2017, 77, 175-186. [CrossRef]

32. Schiff, P.B.; Horwitz, S.B. Taxol stabilizes microtubules in mouse fibroblast cells. Proc. Natl. Acad. Sci. USA 1980, 77, 1561-1565. [CrossRef] [PubMed]

33. Brauer, P.M.; Zheng, Y.; Evans, M.D.; Dominguez-Brauer, C.; Peehl, D.M.; Tyner, A.L. The alternative splice variant of protein tyrosine kinase 6 negatively regulates growth and enhances ptk6-mediated inhibition of beta-catenin. PLoS ONE 2011, 6, e14789. [CrossRef] [PubMed] 